\title{
Article
}

\section{Dual Pharmacological Inhibition of IRAK1 and IRAK4 Pre- vents LPS Induced Monocyte Adhesion to Endothelial Cells}

\author{
Xiumei Wu ${ }^{1 \#}$, Mengyun Xu ${ }^{2 \#, ~ Y u j i e ~ L i u ~}{ }^{2}$, Sihui Luo ${ }^{2}$, Xueying Zheng ${ }^{2}$, Suowen $\mathrm{Xu}^{2, *}$, Jianping Weng ${ }^{1,2 *}$ \\ 1 Department of Endocrinology and Metabolism, Guangdong Provincial Key Laboratory of Diabetology, The \\ Third Affiliated Hospital of Sun Yat-sen University, 510000, Guangzhou, China; wuxm6@sysu.edu.cn \\ (X.W.); wengjp@ustc.edu.cn (J.W.) \\ 2 Institute of Endocrine and Metabolic Diseases, The First Affiliated Hospital of USTC, Division of Life Sci- \\ ences and Medicine, University of Science and Technology of China, 230001, Hefei, China; xumeng- \\ yun0422@163.com (M.X.); liuyujie@ustc.edu.cn (Y.L.); luosihui@ustc.edu.cn (S.L.); lxyzheng@ustc.edu.cn \\ (X.Z.); sxu1984@ustc.edu.cn (S.X.); wengjp@ustc.edu.cn (J.W.) \\ * Correspondence: sxu1984@ustc.edu.cn (S.X.); wengjp@ustc.edu.cn (J.W.) \\ \# These authors contributed equally to this work.
}

\begin{abstract}
Inflammation associated endothelial dysfunction represents a pivotal contributor to atherosclerosis. Increasingly evidence has demonstrated that interleukin 1 receptor (IL1-R) / toll-like receptor (TLR) signaling participated in the development of atherosclerosis. Previous studies indicated the therapeutic potential of anti-inflammatory therapy in anti-atherosclerosis. The present study examined the effect of IL-1R-associated kinase 1 and 4 inhibitors (IRAK1/4i) in regulating endothelial dysfunction. IRAK1/4i showed little endothelial toxicity at concentrations from 1 to 10 $\mu \mathrm{M}$. Inhibition of IRAK1/4 alleviated endothelial activation induced by LPS in vitro evidenced by attenuated monocyte adhesion to the endothelium. Mechanistically, blockade of IRAK1/4 ameliorated the transcriptional activity of NF- $\kappa$ B. Taken together, our findings demonstrated that dual inhibition of IRAK1 and IRAK4 attenuates endothelial dysfunction, suggesting pharmaceutical inhibition of IRAK1/4 might be a potential strategy to combat endothelial dysfunction and atherosclerosis.
\end{abstract}

Keywords: IRAK1; IRAK4; endothelial cells; inflammation; cardiovascular disease

\section{Introduction}

Atherosclerosis is a chronic arterial inflammatory disease driven by endothelial dysfunction and lipid deposition[1]. Hyperlipidemia induced the activation of endothelial cells and cholesterol crystal accumulation, thereby promoting atherothrombosis[2]. Currently, several therapeutic agents have been developed to treat cardiovascular disease (CVD) by targeting lipid metabolism, such as statins. Statins are lipid-lowering medications that inhibit 3-hydroxy-methylglutaryl coenzyme A (HMG-CoA) reductase in cholesterol synthesis in the liver. Despite practical anti-atherogenic effects of statins, many patients were statin-resistant and living with residual inflammatory risk, indicated by high high-sensitivity C-reactive protein levels[3], which might induce life-threatening atherosclerotic events. Therefore, it is essential to develop a novel pharmacologically therapeutic target for vascular disease, reducing residual inflammatory risk in patients receiving standard lipid-lowering therapies.

It is generally acknowledged that inflammation plays an essential role in cardiovascular disease progression[4]. Emerging clinical evidence has revealed the additional role of anti-inflammatory therapy in improving cardiovascular outcomes independent of lipid-lowering. In the CANTOS study[5], specific targeting of interleukin-1 $\beta$ could markedly reduce the rate of recurrent cardiovascular events. The COLCOT study[6] and the LoDoCo2 study[7-8] indicated that a low dose of colchicine decreased cardiovascular 
events in patients with myocardial infarction and with chronic coronary disease, respectively.

Interleukin-1 receptor associated kinase (IRAK, including IRAK1, IRAK2, IRAK3, and IRAK4)[9-10] is a family of serine/threonine kinases involved in toll-like receptor (TLR) and interleukin-1 receptor (IL-1R) signaling pathways against invading pathogen in innate immune response[11-13]. When TLR was activated, myeloid differentiation primary response protein (MyD88) recruited IRAK4 to form myddosome[14], which endogenously recruited and phosphorylated IRAK1[15]. The myddosome complex subsequently bound to tumor necrosis factor receptor-associated factor 6 (TRAF6) and eventually activated the nuclear factor- $\kappa \mathrm{B}(\mathrm{NF}-\kappa \mathrm{B})$ signaling pathway[16-17]. Imbalance in these pathways is recognized to drive diverse pathophysiologies, and recent researcheshave demonstrated the role of IRAKs in cardiovascular and inflammatory diseases[18-20]. Functional deficiency of IRAK1 protected ApoE-/- mice against high-fat diet (HFD)-induced atherosclerosis[21]. Lack of IRAK4 alleviated the development of atherothrombosis

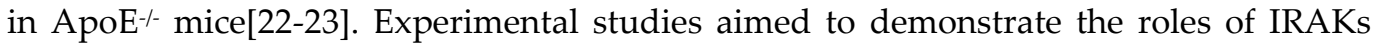
mostly relied on methods such as genetic ablation[22-23] or endogenous down-regulation of IRAK expression[24]. Optimized medicinal chemistry for pharmacologic inhibition of IRAK1 and IRAK4 kinase activity is of great interest in cardiovascular medicine and might be exploited as a potential therapeutic agent for atherosclerosis. Endothelial injury and dysfunction triggered the early development of atherosclerosis, and it represents a therapeutic target for anti-atherogenic effects. However, there was no evidence regarding whether dual pharmacological inhibition of IRAK1/4 inhibitors (IRAK1/4i) protects against endothelial dysfunction in atherosclerosis. Therefore, the present study aimed to examine the role of IRAK1/4 inhibition in regulating LPS elicited dysfunction of human endothelial cells.

\section{Materials and Methods}

\subsection{Isolation and Culture of HUVECs}

Human umbilical vein endothelial cells (HUVECs) were isolated[25] from fresh umbilical cords collected from normal pregnant patients undergoing delivery in the first affiliated hospital of the University of Science and Technology of China (USTC) with written informed consent. The study was approved by institutional review board (IRB) of USTC [2020-ky013]. The cord was cut on both ends, and the umbilical vein was then flushed using PBS several times to eliminate the clots with a $0.5 \mathrm{G}$ needle and syringe. With oneend being clamped with a hemostat, the vein was cannulated and filled with $1 \mathrm{mg} / \mathrm{ml} \mathrm{Col-}$ lagenase I (\#17100-017; Gibco) in McCoy's 5A medium (\#16600-082; Gibco), followed by the other end being clamped. Then, the entire cord was incubated at $37^{\circ} \mathrm{C}$ for 45 minutes, during which gentle massage was performed every 15 minutes. After that, the collagenase digestion solution was collected and the vein was flushed with medium several times to collect all the remaining solution. After the centrifugation at $1400 \mathrm{rpm}$ for 8 minutes, the pellet was resuspended with endothelial cell growth medium (ECM; \#1001; ScienCell) supplemented with 5\% FBS, low serum growth supplements and antibiotics (\#1001; ScienCell). HUVECs were cultured on dishes pre-coated with 0.1\% gelatin (\#A609764-0500; Sangon, Shanghai, China), and maintained in an incubator with $5 \% \mathrm{CO} 2$ at $37^{\circ} \mathrm{C}$. HUVECs with a passage number of less than six-passage were used for the experiments. HUVECs were seeded into 12-well plates or 96-well plates after reaching sub-confluence. In the present study, HUVECs cells were pre-incubated with IRAK1/4i or vehicle overnight, followed by 6-hour treatment with $1 \mathrm{ug} / \mathrm{ml}$ lipopolysaccharide (LPS; \#089M4043V; Sigma). IRAK1/4 inhibitor (\#509093-47-4; Topscience, China) was dissolved in DMSO and diluted with the medium before use.

\subsection{RNA Extraction, Reverse Transcription and RT-qPCR}


Total RNA was extracted with RNeasy MiniKit (\#74106; Qiagen, Germany) according to the specification. Reverse transcription was conducted using the PrimeScript RT reagent Kit (\#RR037B; TAKARA, Japan) after RNA isolation and quantitation. Real timequantitative polymerase chain reaction (RT-qPCR) experiments were performed using SYBR Green RT-PCR Kit (\#RR420A; TAKARA, Japan) and The LightCycler $® 2.0$ Instrument (Roche Diagnostics GmbH, Mannheim, Germany) following the manufacturer's instructions. The sequence of primers for RT-qPCR was listed in Appendix Table 1.

\subsection{Western Blot}

After treatment and incubation, HUVECs were washed twice with PBS and lysed with 1Xloading buffer. Boiled proteins were loaded in 10\% SDS/PAGE gels and then transferred to the NC membranes. The membranes were blocked using PBS-diluted blocking buffer (\#927-60001; Li-Cor) for one hour, followed by incubation with specific antibodies (listed in Appendix Table 1) overnight, and then incubated with IRD680 (\#C90910-21; LiCor) or IRD800 (\#C91211-03; Li-Cor) secondary antibodies for one hour. After the final wash with TBST, we visualized the membranes with the Odyssey CLx system (Li-Cor, USA).

\subsection{Cell Viability Assay}

HUVECs were seeded into 96-well plates at $1 \times 10^{4}$ cells $/ \mathrm{cm}^{2}$ in $100 \mu \mathrm{L}$ medium. The cell counting kit-8 assay (\#BS354A; Biosharp) was used for cell viability assessment at 24 hours after incubation with IRAK1/4i at $0,1,2.5,5$, and $10 \mu \mathrm{M}$, following the manufacturer's guideline. The plate was measured at an absorbance of $450 \mathrm{~nm}$ using a microplate reader (Molecular Devices, iD3).

\subsection{THP1 Culture and Monocyte Adhesion Assay}

THP1 monocytes (human monocytic leukemia cell line) (\#TIB-202; ATCC) were cultured in RPMI 1640 medium (\#61870036; Gibco) supplemented with $10 \%$ fetal bovine serum (\#10100; Gibco) and 1\% penicillin-streptomycin (\#15140-122; Gibco). THP1 cells were grown at $100 \%$ humidity, $5 \% \mathrm{CO} 2$, and $37^{\circ} \mathrm{C}$. HUVECs were seeded on 12 -well plates (1 $\left.\times 10^{4} \mathrm{cells} / \mathrm{cm}^{2}\right)$ and were treated with IRAK1/4i or vehicle overnight. LPS $(1 \mu \mathrm{g} / \mathrm{ml})$ or LPS $(1 \mu \mathrm{g} / \mathrm{ml})$ plus IL-1 $\beta(10 \mathrm{ng} / \mathrm{ml})(\# ;)$ treatment was performed for 6 hours to activate HUVECs. Thereafter, THP1 cells were seeded onto the monolayer HUVECs at $1 \times 10^{5}$ cells/well and incubated at $37^{\circ} \mathrm{C}$ for 30 minutes. Non-adherent monocytes were removed by gentle washing with ECM medium thrice. Adherent monocytes were visualized using a microscope (\#), and three photos were taken from each well. The number of adherent monocytes was calculated by counting, and the average value was calculated and presented.

\subsection{Luciferase Reporter Assay}

HUVECs were plated into 12-well plates at $1 \times 10^{4}$ cells $/ \mathrm{cm}^{2}$ before transfection. The NF- $\kappa$ B-luciferase activity was determined. In brief, HUVECs were transfected with Ad-NF-kB-luc (Vector Biolab Inc., M.O.I.=1) for 48 hours. HUVECs were then treated with IRAK1/4i or vehicle overnight, followed by incubation of LPS $(1 \mu \mathrm{g} / \mathrm{ml})$ for 3 hours. The cells were harvested with lysis buffer, and dual luciferase activity was measured using a microplate reader (Molecular Devices, iD3).

\subsection{Statistical Analyses}

Data were expressed as mean \pm standard deviation. Unpaired $t$-test was performed for comparison between two groups. We conducted one-way Analysis of Variance (ANOVA) for comparison among multigroup and the Bonferroni correction for multiple comparisons. Data analysis was conducted with GraphPad Prism 8.0. P-values less than 0.05 were considered to be statistically significant. 


\section{Results}

\subsection{Effect of IRAK1/4i on Endothelial Cell Viability.}

IRAK family includes a centric kinase domain and a highly conserved death domain. Though IRAK1 and IRAK4 share only 31\% sequence in similarity, they shared many comparabilities in their inhibitory binding pockets, a typical ATP binding site[26]. IRAK1/4i is a dual inhibitor of IRAK1 and IRAK4. The structure of the IRAK1/4i was displayed as in Figure 1A. We first examined the effect of IRAK1/4i on endothelial cell viability. CCK8 assay was conducted to evaluate cellular growth and viability. IRAK1/4i showed no signs of cytotoxicity in HUVECs after incubation for 24 hours from the concentration from $0 \mu \mathrm{M}$ to $10 \mu \mathrm{M}$ (Figure 1B). Taken together, IRAK1/4i was not cytotoxic in HUVECs with concentrations tested and provided a possibility for further therapeutic inhibition.

A

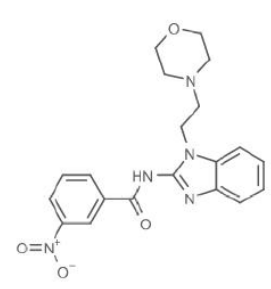

B

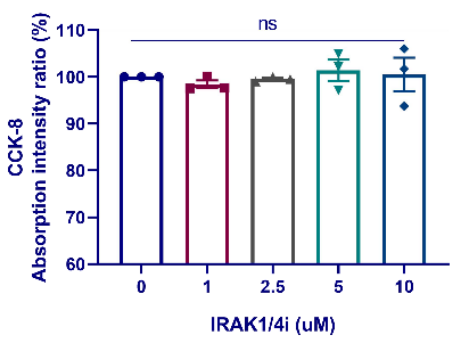

Figure 1. Effect of IRAK1/4 inhibitor on endothelial cell viability.

A, The chemical structure of IRAK1/4 inhibitor. B, The cell viability of HUVECs treated with 0, 1, 2.55 , and $10 \mu \mathrm{M}$ IRAK1/4i for 24 hours ( $\mathrm{n}=3$ biological replicates). Data were represented as the mean \pm SEM. Statistical significance was analyzed using one-way ANOVA. IRAK1/4, Interleukin 1 receptor-associated kinase-1/4; HUVECs, human umbilical vein endothelial cells.

\subsection{Inhibition of IRAK1/4 Alleviated Endothelial Activation Induced by LPS in vitro.}

The endothelium is of great importance in regulating vascular inflammation. The activation of endothelial cells upon inflammatory stimulation will up-regulate two essential adhesion molecules, intercellular adhesion molecule-1 (ICAM-1) and vascular cell adhesion molecule-1 (VCAM-1). Next, we evaluated the effect of IRAK1/4i in the activation of HUVEC induced by LPS. Treatment of LPS in HUVECsincreased the mRNA abundance of VCAM-1 and ICAM-1, but IRAK1/4i reduced this response in a dose-independent manner (Figure 2A-B). Selectin E (SELE) is also a critical adhesion molecule in activated endothelium by promoting leukocyte recruitment[27]. Challenge of HUVECs with LPS up-regulated the mRNA level of SELE, and this induction was decreased by IRAK1/4i treatment (Figure 2C). Accordingly, LPS induced the protein abundance of ICAM-1 and VCAM-1 in HUVECs, which was markedly reduced by IRAK1/4i (Figure 2D). Taken together, inhibition of IRAK1/4 alleviated the activation in HUVECs induced by LPS in a dose-independent manner. 

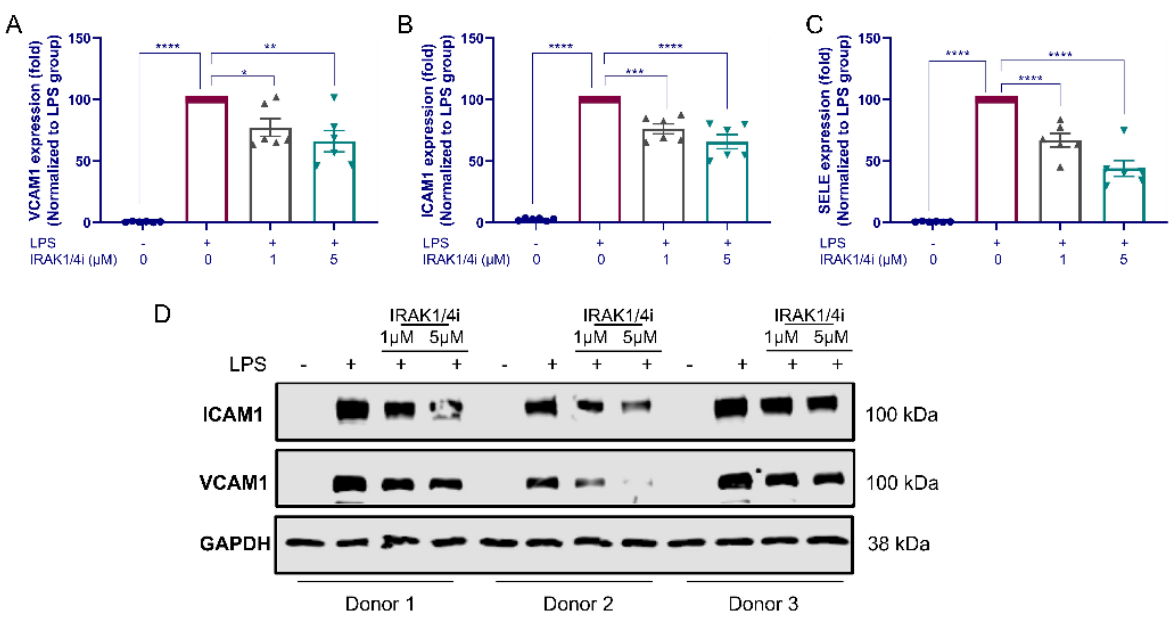

Figure 2. IRAK1/4 inhibitor decreased the expression of adhesion molecules in cultured HUVECs.

HUVECs were pre-incubated with vehicle (DMSO) or IRAK1/4i (1 $\mu \mathrm{M}$ and $5 \mu \mathrm{M})$ overnight and incubated with LPS $(1 \mu \mathrm{g} / \mathrm{ml})$ for 6 hours. qRT-PCR was then used to measure the mRNA level of VCAM1 (A), ICAM1 (B) and SELE (C) in HUVECs induced by LPS ( $\mathrm{n}=6$ biological replicates). For A-C, GAPDH was used as the internal reference. Western blots were then conducted to measure the expression level of VCAM1 and ICAM1 (D) ( $\mathrm{n}=3$ biological replicates). In total, six separate experiments were performed, and panel D showed three of them. Statistical significance was analyzed using one-way ANOVA and the Bonferroni correction. Data were represented as the mean \pm SEM. ${ }^{*} \mathrm{P}<0.05,{ }^{* *} \mathrm{P}<0.01,{ }^{* * *} \mathrm{P}<0.001,{ }^{* * *} \mathrm{P}<0.0001$ vs. LPS group. IRAK1/4, Interleukin 1 receptorassociated kinase-1/4; HUVECs, human umbilical vein endothelial cells; LPS, lipopolysaccharide.

\subsection{IRAK1/4 Inhibition Attenuated Monocyte Adhesion to the Endothelium.}

The recruitment and adhesion of monocytes to the endothelial cells occur in the early stage of vascular inflammation. Next, we asked whether IRAK1/4i would ameliorate monocyte adhesion to activated endothelial cells. As shown in Figure 3A-B, LPS stimulated monocyte adhesion to endothelial cells. However, IRAK1/4i treatment leads to decreased adhesion of monocytes to HUVECs. IRAK1 and IRAK4 act as essential regulators in TLR and IL-1 $\beta$ pathway, thus we conducted an additional stimulation of LPS plus IL-1 $\beta$. Challenge of LPS plus IL-1 $\beta$ in HUVECs induced more obvious monocyte adhesion, and inhibition of IRAK1/4 alleviated the adhesion dose-independently. Thus, pharmacological blockade of IRAK1/4 reduced monocyte adhesion to activated endothelium.
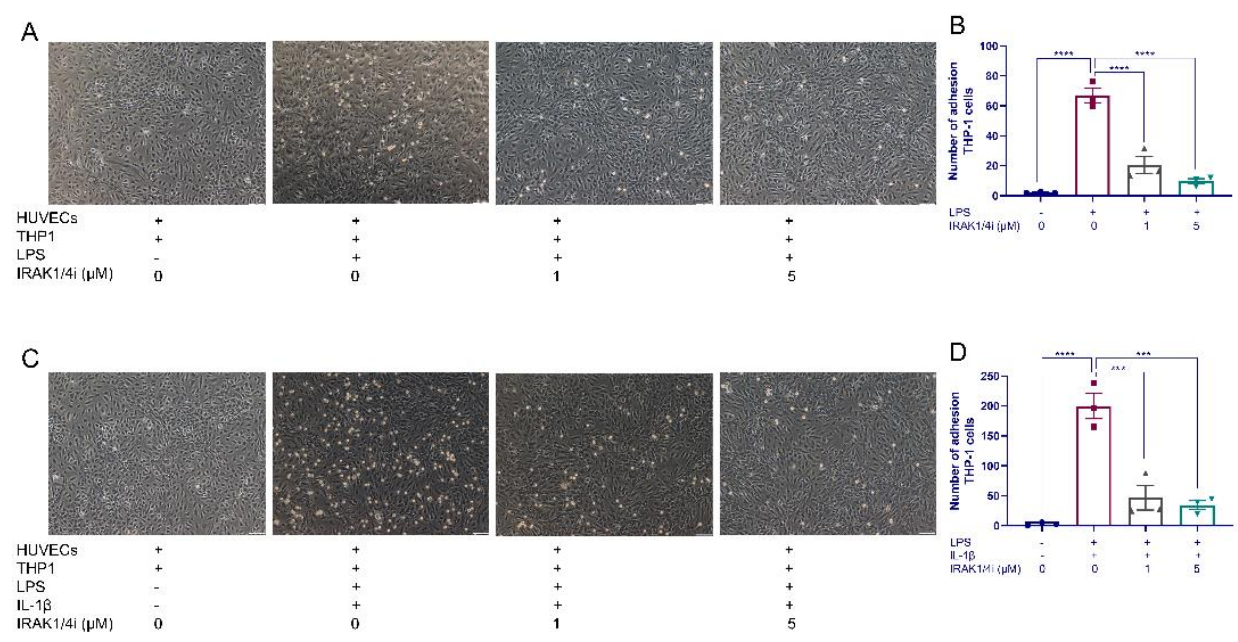

Figure 3. IRAK1/4 inhibitor attenuated monocyte adhesion to endothelial cells. 
HUVE cells were pre-incubated with vehicle (DMSO) or IRAK1/4i (1 $\mu \mathrm{M}$ and $5 \mu \mathrm{M})$ overnight, and treated with LPS $(1 \mu \mathrm{g} / \mathrm{ml})(\mathbf{A})$ or LPS $(1 \mu \mathrm{g} / \mathrm{ml})$ plus IL-1 $\beta(10 \mathrm{ng} / \mathrm{ml})(\mathbf{B})$ for 6 hours, followed by THP1 cell adhesion assay, $n=3$ biological replicates. Statistical significance was analyzed using one-way ANOVA and the Bonferroni correction. Data were represented as the mean \pm SEM. ${ }^{* * *} \mathrm{P}$ $<0.001,{ }^{* * * *} \mathrm{P}<0.0001$ vs. LPS group. IRAK1/4, Interleukin 1 receptor-associated kinase-1/4; HUVECs, Human umbilical vein endothelial cells; LPS, lipopolysaccharide.

\subsection{Blockade of IRAK1/4 Ameliorated the Transcriptional Activity of NF- $\kappa B$.}

We finally explored the mechanism of IRAK1/4 inhibition on the endothelial inflammatory response. NF- $\kappa \mathrm{B}$ activation in endothelial cells is fundamental to inflammation initiation and adhesion molecule induction. Thus, we investigated the role of the NF- $\kappa \mathrm{B}$. As displayed in Figure 4, LPS obviously upregulated the transcriptional activity of NF$\kappa \mathrm{B}$, but this induction was reduced by IRAK1/4 suppression in a dose-independent manner. Thus, blockade of IRAK1/4 ameliorated the transcriptional activity of NF- $\kappa$ B by LPS stimulation.

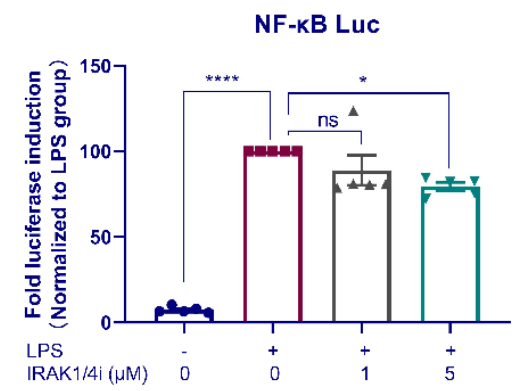

Figure 4. IRAK1/4 inhibitor reduced the transcriptional activity of NF-KB.

HUVECs were pre-incubated with vehicle (DMSO) or IRAK1/4i ( $1 \mu \mathrm{M}$ and $5 \mu \mathrm{M})$ overnight, and treated with LPS $(1 \mu \mathrm{g} / \mathrm{ml})$ for 3 hours. Luciferase reporter assay was then preformed to measure the transcriptional activity of NF- $\kappa \mathrm{B}, \mathrm{n}=5$ biological replicates. Statistical significance was analyzed using one-way ANOVA and the Bonferroni correction. Data were represented as the mean \pm SEM. ${ }^{*} \mathrm{P}<0.05,{ }^{* *} \mathrm{P}<0.01,{ }^{* * *} \mathrm{P}<0.001,{ }^{* * * *} \mathrm{P}<0.0001$ vs. LPS group. IRAK1/4, Interleukin 1 receptorassociated kinase; HUVECs, human umbilical vein endothelial cells; LPS, lipopolysaccharide.

\section{Discussion}

TLR/IL-1R signaling and NF- $\kappa$ B activation participate in the early stage of atherosclerosis[28-29]. In the current study, we examined the role of IRAK1/4 in endothelial function by using a dual inhibitor of IRAK1 and IRAK4. We demonstrated that suppression of IRAK1 and IRAK4 alleviated endothelial activation, attenuated monocyte adhesion, and ameliorated the transcriptional activity of NF- $\kappa B$ in HUVECs. The results indicated the critical role of IRAK1/4 in endothelial dysfunction and inflammation.

IRAK1 and IRAK4, two key kinases in TLR-MyD88 pathway, play essential roles in the progression of atherosclerosis[30]. In the early stage of arteriosclerosis, endothelial damage, increased endothelial permeability, upregulation of adherent molecules, monocyte adhesion, and transmigration represent important hallmarks of endothelial dysfunction[31]. Abnormal activation in the endothelium played an essential role in atherosclerotic pathogenesis. It is well recognized that enhanced oxidative stress, induced by exogenous and endogenous LPS, was a critical trigger in endothelial dysfunction[32]. IRAK1/4 inhibition, which was demonstrated to suppress LPS-induced endothelial dysfunction in 
the present results, provided the rationale and potential for future clinical trials to prevent the pathological development in CVD patients with residual inflammatory risks.

Previous studies of IRAK1/4 inhibition were mainly derived from genetic knockout or knockdown systems rather than small-molecule inhibitors. Existing researches have reported the method of IRAK knockout mice[33-34], IRAK kinase mutation knock-in mice[23,35], knockdown microRNA[36-37], small interfering RNA[38-39], and short hairpin RNA[40] to abolish the function of IRAK1 and IRAK4. Available chemical inhibitors were increasingly demanding and held great therapeutic potential.

Although IRAK1 and IRAK4 inhibition have not been evaluated in clinical settings, the positive results in the recent CANTOS study regarding to IL- $1 \beta$ suppression in atherosclerosis provide the impetus to test this concept whether IRAK1/4 pharmaceutical inhibition is therapeutically beneficial. In the CANTOS trial[5], canakinumab, the anti-IL-1 $\beta$ antibody, reduced the rate of recurring cardiovascular death in patients with myocardial infarction (MI) and high-sensitivity CRP levels. Besides, treatment of low-dose colchicine in the COLCOT trial[6] and the LODOCO2 trial[8] also showed a significant reduction in cardiovascular events in colchicine-treated patients with previous MI or chronic coronary disease. The successful outcomes of these trials reinforced the concept that targeting IRAK1/4 may lower residual inflammatory risk in patients resistant to statin therapy. However, a higher incidence of sepsis and fatal infection was reported in the IL- $1 \beta$ blockade group[5], which might indicate the essence and demand of a safer pharmacological target against inflammation in atherothrombosis. Inhibition of IRAK1/4 partially diminished the TLR/IL-1R signaling and NF- $\kappa B$ activation. An incomplete blockade of the innate immune response induced by an alternative pathway might help foster protective defense. Additional surveys are required to elucidate the potential effect of IRAK1/4 suppressionon vascular inflammation and atherosclerosis in vivo. Taken together, our study offers direct evidence that pharmacological inhibition of IRAK1/4 will be a promising therapy for treating atherosclerosis.

\section{Conclusions}

Suppression of IRAK1 and IRAK4 alleviated endothelial activation induced by LPS in vitro by attenuated monocyte adhesion to the endothelium. Inhibition of IRAK1/4 ameliorated the transcriptional activity of NF- $\mathrm{B}$. Taken together, dual inhibition of IRAK1 and IRAK4 attenuates endothelial dysfunction, suggesting pharmaceutical inhibition of IRAK1/4 might be a potential strategy to combat endothelial dysfunction and atherosclerosis.

\section{Supplementary Materials: Appendix Table $1 .$.}

Author Contributions: Conceptualization, JW and SX; validation, XW and MX; investigation, XW and $\mathrm{MX}$; resources, $\mathrm{YL}$; data curation, $\mathrm{XW}$ and $\mathrm{MX}$; writing-original draft preparation, $\mathrm{XW}$ and MX; writing - review and editing, JW and SX; visualization, XW and MX; supervision, XZ, SL, JW and SX; project administration, XZ, SL, JW and SX; funding acquisition, XZ, SL, JW and SX. All authors have read and agreed to the published version of the manuscript.

Funding: This study was funded by grants from National Natural Science Foundation of China [Grant Nos. 81941022 to JW, 81530025 to JW, 82070464 to SX], Strategic Priority Research Program of Chinese Academy of Sciences [Grant No. XDB38010100 to JW]. This work was also supported by Program for Innovative Research Team of The First Affiliated Hospital of USTC (to JW), Local Innovative and Research Teams Project of Guangdong Pearl River Talents Program [2017BT01S131], Natural Science Foundation of Anhui Province [Grant No. 006223066002 to SL] as well as a special fund from Hefei Comprehensive National Science Center.

Institutional Review Board Statement: The study was approved by institutional review board (IRB) of USTC [2020-ky013].

Informed Consent Statement: Informed consent was obtained from all subjects involved in the study.

Data Availability Statement: Not applicable. 


\begin{abstract}
Acknowledgments: We want to thank the pregnant women for donating the umbilical cords. We would like to thank satff in the Department of Endocrinology and Metabolism of The Third Affiliated Hospital of Sun Yat-sen University and The First Affiliated Hospital of USTC of Division of Life Sciences and Medicine of University of Science and Technology of China for technical assistance.
\end{abstract}

Conflicts of Interest: The authors declare that they have no competing interests.

Appendix Table 1 Primers and antibodies

\begin{tabular}{ll}
\hline \multicolumn{1}{c}{ Primers } & \multicolumn{1}{c}{ Sequence } \\
\hline Human-GAPDH-F & CAAGAGCACAAGAGGAAGAGAG \\
Human-GAPDH-R & CTACATGGCAACTGTGAGGAG \\
Human-VCAM1-F & GGAGCTCTACTCATTCCCTAGA \\
Human-VCAM1-R & CTAGGAACCTTGCAGCTTACA \\
Human-ICAM1-F & CCTCAGCACGTACCTCTATAAC \\
Human-ICAM1-R & GGCTTGTGTGTTCGGTTTC \\
Human-SELE-F & GTGTATGTCCTCTGGAGAATGG \\
Human-SELE-R & GAACCCATTGGCTGGATTTG \\
\multicolumn{1}{c}{ Antibody } & Company \\
Human-VCAM1 & \# 383323, Zenbio, China \\
Human-ICAM1 & \# 380990, Zenbio, China \\
Human-GAPDH & \# ab8245, Abcam \\
\hline
\end{tabular}

\title{
References
}

1. Libby, P.; Ridker, P. M.; Hansson, G. K., Inflammation in atherosclerosis: from pathophysiology to practice. J. Am. Coll. Cardiol. 2009, 54, 2129-38 https://doi.org/10.1016/j.jacc.2009.09.009.

2. Baumer, Y.; McCurdy, S.; Weatherby, T. M.; Mehta, N. N.; Halbherr, S.; Halbherr, P.; Yamazaki, N.; Boisvert, W. A., Hyperlipidemia-induced cholesterol crystal production by endothelial cells promotes atherogenesis. Nat Commun 2017, 8 , 1129 https://doi.org/10.1038/s41467-017-01186-z.

3. Ridker, P. M., How Common Is Residual Inflammatory Risk? Circul. Res. 2017, 120, 617-619 https://doi.org/10.1161/circresaha.116.310527.

4. Libby, P.; Buring, J. E.; Badimon, L.; Hansson, G. K.; Deanfield, J.; Bittencourt, M. S.; Tokgözoğlu, L.; Lewis, E. F., Atherosclerosis. Nat Rev Dis Primers 2019, 5, 56 https://doi.org/10.1038/s41572-019-0106-z.

5. Ridker, P. M.; Everett, B. M.; Thuren, T.; MacFadyen, J. G.; Chang, W. H.; Ballantyne, C.; Fonseca, F.; Nicolau, J.; Koenig, W.; Anker, S. D.; Kastelein, J. J. P.; Cornel, J. H.; Pais, P.; Pella, D.; Genest, J.; Cifkova, R.; Lorenzatti, A.; Forster, T.; Kobalava, Z.; Vida-Simiti, L.; Flather, M.; Shimokawa, H.; Ogawa, H.; Dellborg, M.; Rossi, P. R. F.; Troquay, R. P. T.; Libby, P.; Glynn, R. J., Antiinflammatory Therapy with Canakinumab for Atherosclerotic Disease. New Engl. J. Med. 2017, 377, 1119-1131 https://doi.org/10.1056/NEJMoa1707914.

6. Tardif, J. C.; Kouz, S.; Waters, D. D.; Bertrand, O. F.; Diaz, R.; Maggioni, A. P.; Pinto, F. J.; Ibrahim, R.; Gamra, H.; Kiwan, G. S.; Berry, C.; López-Sendón, J.; Ostadal, P.; Koenig, W.; Angoulvant, D.; Grégoire, J. C.; Lavoie, M. A.; Dubé, M. P.; Rhainds, D.; Provencher, M.; Blondeau, L.; Orfanos, A.; L'Allier, P. L.; Guertin, M. C.; Roubille, F., Efficacy and Safety of Low-Dose Colchicine after Myocardial Infarction. New Engl. J. Med. 2019, 381, 2497-2505 https://doi.org/10.1056/NEJMoa1912388.

7. Nidorf, S. M.; Eikelboom, J. W.; Budgeon, C. A.; Thompson, P. L., Low-dose colchicine for secondary prevention of cardiovascular disease. J. Am. Coll. Cardiol. 2013, 61, 404-410 https://doi.org/10.1016/j.jacc.2012.10.027.

8. Nidorf, S. M.; Fiolet, A. T. L.; Mosterd, A.; Eikelboom, J. W.; Schut, A.; Opstal, T. S. J.; The, S. H. K.; Xu, X. F.; Ireland, M. A.; Lenderink, T.; Latchem, D.; Hoogslag, P.; Jerzewski, A.; Nierop, P.; Whelan, A.; Hendriks, R.; Swart, H.; Schaap, J.; Kuijper, A. F. M.; van Hessen, M. W. J.; Saklani, P.; Tan, I.; Thompson, A. G.; Morton, A.; Judkins, C.; Bax, W. A.; Dirksen, M.; Alings, M.; 
Hankey, G. J.; Budgeon, C. A.; Tijssen, J. G. P.; Cornel, J. H.; Thompson, P. L., Colchicine in Patients with Chronic Coronary Disease. New Engl. J. Med. 2020, 383, 1838-1847 https://doi.org/10.1056/NEJMoa2021372.

9. Flannery, S.; Bowie, A. G., The interleukin-1 receptor-associated kinases: critical regulators of innate immune signalling. Biochem. Pharmacol. 2010, 80, 1981-91 https://doi.org/10.1016/j.bcp.2010.06.020.

10. Cao, Z.; Henzel, W. J.; Gao, X., IRAK: a kinase associated with the interleukin-1 receptor. Science 1996, 271, 1128-31 https://doi.org/10.1126/science.271.5252.1128.

11. Alfaidi, M.; Acosta, C. H.; Wang, D.; Traylor, J. G.; Orr, A. W., Selective role of Nck1 in atherogenic inflammation and plaque formation. J. Clin. Invest. 2020, 130, 4331-4347 https://doi.org/10.1172/jci135552.

12. Cohen, P., The TLR and IL-1 signalling network at a glance. J. Cell Sci. 2014, 127, 2383-90 https://doi.org/10.1242/jcs.149831.

13. Jain, A.; Kaczanowska, S.; Davila, E., IL-1 Receptor-Associated Kinase Signaling and Its Role in Inflammation, Cancer Progression, and Therapy Resistance. Front. Immunol. 2014, 5, 553 https://doi.org/10.3389/fimmu.2014.00553.

14. Kawai, T.; Akira, S., The role of pattern-recognition receptors in innate immunity: update on Toll-like receptors. Nat. Immunol. 2010, 11, 373-84 https://doi.org/10.1038/ni.1863.

15. Bhaumik, D.; Scott, G. K.; Schokrpur, S.; Patil, C. K.; Campisi, J.; Benz, C. C., Expression of microRNA-146 suppresses NFkappaB activity with reduction of metastatic potential in breast cancer cells. Oncogene 2008, 27, 5643-7 https://doi.org/10.1038/onc.2008.171.

16. Conze, D. B.; Wu, C. J.; Thomas, J. A.; Landstrom, A.; Ashwell, J. D., Lys63-linked polyubiquitination of IRAK-1 is required for interleukin-1 receptor- and toll-like receptor-mediated NF-kappaB activation. Mol. Cell. Biol. 2008, 28, 3538-47 https://doi.org/10.1128/mcb.02098-07.

17. Swantek, J. L.; Tsen, M. F.; Cobb, M. H.; Thomas, J. A., IL-1 receptor-associated kinase modulates host responsiveness to endotoxin. J. Immunol. 2000, 164, 4301-6 https://doi.org/10.4049/jimmunol.164.8.4301.

18. Lakoski, S. G.; Li, L.; Langefeld, C. D.; Liu, Y.; Howard, T. D.; Brosnihan, K. B.; Xu, J.; Bowden, D. W.; Herrington, D. M., The association between innate immunity gene (IRAK1) and C-reactive protein in the Diabetes Heart Study. Exp. Mol. Pathol. 2007, 82, 280-3 https://doi.org/10.1016/j.yexmp.2007.02.002.

19. Gao, M.; Wang, X.; Zhang, X.; Ha, T.; Ma, H.; Liu, L.; Kalbfleisch, J. H.; Gao, X.; Kao, R. L.; Williams, D. L.; Li, C., Attenuation of Cardiac Dysfunction in Polymicrobial Sepsis by MicroRNA-146a Is Mediated via Targeting of IRAK1 and TRAF6 Expression. J. Immunol. 2015, 195, 672-82 https://doi.org/10.4049/jimmunol.1403155.

20. Toubiana, J.; Courtine, E.; Pène, F.; Viallon, V.; Asfar, P.; Daubin, C.; Rousseau, C.; Chenot, C.; Ouaaz, F.; Grimaldi, D.; Cariou, A.; Chiche, J. D.; Mira, J. P., IRAK1 functional genetic variant affects severity of septic shock. Crit. Care Med. 2010, 38, 2287-94 https://doi.org/10.1097/CCM.0b013e3181f9f9c7.

21. Wang, D.; Fasciano, S.; Li, L., The interleukin-1 receptor associated kinase 1 contributes to the regulation of NFAT. Mol. Immunol. 2008, 45, 3902-8 https://doi.org/10.1016/j.molimm.2008.06.023.

22. Rekhter, M.; Staschke, K.; Estridge, T.; Rutherford, P.; Jackson, N.; Gifford-Moore, D.; Foxworthy, P.; Reidy, C.; Huang, X. D.; Kalbfleisch, M.; Hui, K.; Kuo, M. S.; Gilmour, R.; Vlahos, C. J., Genetic ablation of IRAK4 kinase activity inhibits vascular lesion formation. Biochem. Biophys. Res. Commun. 2008, 367, 642-8 https://doi.org/10.1016/j.bbrc.2007.12.186.

23. Kim, T. W.; Febbraio, M.; Robinet, P.; Dugar, B.; Greene, D.; Cerny, A.; Latz, E.; Gilmour, R.; Staschke, K.; Chisolm, G.; Fox, P. L.; DiCorleto, P. E.; Smith, J. D.; Li, X., The critical role of IL-1 receptor-associated kinase 4-mediated NF- $\kappa$ B activation in modified low-density lipoprotein-induced inflammatory gene expression and atherosclerosis. J. Immunol. 2011, 186, 2871-80 https://doi.org/10.4049/jimmunol.1002242.

24. Taganov, K. D.; Boldin, M. P.; Chang, K. J.; Baltimore, D., NF-kappaB-dependent induction of microRNA miR-146, an inhibitor targeted to signaling proteins of innate immune responses. Proc. Natl. Acad. Sci. U. S. A. 2006, 103, 12481-6 https://doi.org/10.1073/pnas.0605298103.

25. Baudin, B.; Bruneel, A.; Bosselut, N.; Vaubourdolle, M., A protocol for isolation and culture of human umbilical vein endothelial cells. Nat. Protoc. 2007, 2, 481-5 https://doi.org/10.1038/nprot.2007.54.

26. Singer, J. W.; Fleischman, A.; Al-Fayoumi, S.; Mascarenhas, J. O.; Yu, Q.; Agarwal, A., Inhibition of interleukin-1 receptor-associated kinase 1 (IRAK1) as a therapeutic strategy. Oncotarget. 2018, 9, 33416-33439 https://doi.org/10.18632/oncotarget.26058.

27. Kansas, G. S., Selectins and their ligands: current concepts and controversies. Blood 1996, 88, 3259-87

28. de Winther, M. P.; Kanters, E.; Kraal, G.; Hofker, M. H., Nuclear factor kappaB signaling in atherogenesis. Arterioscler Thromb Vasc. 2005, 25, 904-14 https://doi.org/10.1161/01.Atv.0000160340.72641.87.

29. Chi, H.; Messas, E.; Levine, R. A.; Graves, D. T.; Amar, S., Interleukin-1 receptor signaling mediates atherosclerosis associated with bacterial exposure and/or a high-fat diet in a murine apolipoprotein E heterozygote model: pharmacotherapeutic implications. Circulation 2004, 110, 1678-85 https://doi.org/10.1161/01.Cir.0000142085.39015.31.

30. Dunne, A.; Carpenter, S.; Brikos, C.; Gray, P.; Strelow, A.; Wesche, H.; Morrice, N.; O'Neill, L. A. J., IRAK1 and IRAK4 promote phosphorylation, ubiquitination, and degradation of MyD88 adaptor-like (Mal). J. Biol. Chem. 2016, 291, 24802 https://doi.org/10.1074/jbc.A109.098137.

31. Esper, R. J.; Nordaby, R. A.; Vilariño, J. O.; Paragano, A.; Cacharrón, J. L.; Machado, R. A., Endothelial dysfunction: a comprehensive appraisal. Cardiovasc. Diabetol. 2006, 5, 4 https://doi.org/10.1186/1475-2840-5-4.

32. Steyers, C. M., 3rd; Miller, F. J., Jr., Endothelial dysfunction in chronic inflammatory diseases. Int. J. Mol. Sci. 2014, 15, 11324-49 https://doi.org/10.3390/ijms150711324.

33. Thomas, J. A.; Allen, J. L.; Tsen, M.; Dubnicoff, T.; Danao, J.; Liao, X. C.; Cao, Z.; Wasserman, S. A., Impaired cytokine signaling in mice lacking the IL-1 receptor-associated kinase. J. Immunol. 1999, 163, 978-84 
34. Sun, X. J.; Kim, S. P.; Zhang, D.; Sun, H.; Cao, Q.; Lu, X.; Ying, Z.; Li, L.; Henry, R. R.; Ciaraldi, T. P.; Taylor, S. I.; Quon, M. J., Deletion of interleukin 1 receptor-associated kinase 1 (Irak1) improves glucose tolerance primarily by increasing insulin sensitivity in skeletal muscle. J. Biol. Chem. 2017, 292, 12339-12350 https://doi.org/10.1074/jbc.M117.779108.

35. Pauls, E.; Nanda, S. K.; Smith, H.; Toth, R.; Arthur, J. S. C.; Cohen, P., Two phases of inflammatory mediator production defined by the study of IRAK2 and IRAK1 knock-in mice. J. Immunol. 2013, 191, 2717-30 https://doi.org/10.4049/jimmunol.1203268.

36. Hung, P. S.; Liu, C. J.; Chou, C. S.; Kao, S. Y.; Yang, C. C.; Chang, K. W.; Chiu, T. H.; Lin, S. C., miR-146a enhances the oncogenicity of oral carcinoma by concomitant targeting of the IRAK1, TRAF6 and NUMB genes. PLoS One 2013, 8, e79926 https://doi.org/10.1371/journal.pone.0079926.

37. Jiang, W.; Kong, L.; Ni, Q.; Lu, Y.; Ding, W.; Liu, G.; Pu, L.; Tang, W.; Kong, L., miR-146a ameliorates liver ischemia/reperfusion injury by suppressing IRAK1 and TRAF6. PLoS One 2014, 9, e101530 https://doi.org/10.1371/journal.pone.0101530.

38. Della Mina, E.; Borghesi, A.; Zhou, H.; Bougarn, S.; Boughorbel, S.; Israel, L.; Meloni, I.; Chrabieh, M.; Ling, Y.; Itan, Y.; Renieri, A.; Mazzucchelli, I.; Basso, S.; Pavone, P.; Falsaperla, R.; Ciccone, R.; Cerbo, R. M.; Stronati, M.; Picard, C.; Zuffardi, O.; Abel, L.; Chaussabel, D.; Marr, N.; Li, X.; Casanova, J. L.; Puel, A., Inherited human IRAK-1 deficiency selectively impairs TLR signaling in fibroblasts. Proc. Natl. Acad. Sci. U. S. A. 2017, 114, E514-e523 https://doi.org/10.1073/pnas.1620139114.

39. Li, M.; Yu, D.; Ni, B.; Hao, F., Interleukin-1 receptor associated kinase 1 is a potential therapeutic target of anti-inflammatory therapy for systemic lupus erythematosus. Mol. Immunol. 2017, 87, 94-101 https://doi.org/10.1016/j.molimm.2017.03.018.

40. Adams, A. K.; Bolanos, L. C.; Dexheimer, P. J.; Karns, R. A.; Aronow, B. J.; Komurov, K.; Jegga, A. G.; Casper, K. A.; Patil, Y. J.; Wilson, K. M.; Starczynowski, D. T.; Wells, S. I., IRAK1 is a novel DEK transcriptional target and is essential for head and neck cancer cell survival. Oncotarget. 2015, 6, 43395-407 https://doi.org/10.18632/oncotarget.6028. 